\title{
Über die eigenartige Wirkung von H-Ionen bei der Bildung von sauren Alkylsulfaten aus den neutralen dureh Wasser im heterogenen System

\author{
von
}

R. Kremann.

Aus dem chemischen Institute der Universität in Graz.

(Mit 1 Textfigur.)

(Vorgelegt in der Sitzung am 16. November 1906.)

Es ist aus der Literatur bekannt, daß die Darstellung von Äthylschwefelsäure und auch von Methylschwefelsäure am einfachsten gelingt, wenn man Diäthylsulfat, beziehungsweise Dimethylsulfat mit warmem Wasser schüttelt. ${ }^{1}$

Diese Darstellungsweise bietet ein hervorstechendes Beispiel für den stufenweisen Verlauf der Abspaltung von Alkyl- · gruppen der Ester einer mehrbasischen Säure und es war daher ein gewisses Interesse, oben erwähnte Reaktion kinetisch messend zu verfolgen.

Bei Temperaturen, bei welchen die Abspaltung der einen Alkylgruppe nach der Gleichung

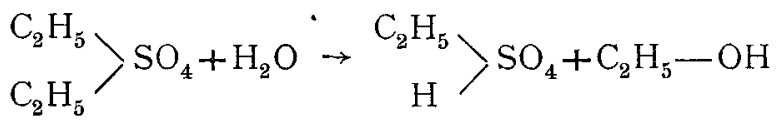

mit einer für die messende Verfolgung des Reaktionsverlaufes geeigneten Geschwindigkeit von statten geht, d. i. bei zirka 30 bis $50^{\circ}$, erfolgt die Zersetzung der Äthylschwefelsäure durch

1 Serullas, Ann. chim. phys., 39, 153. - Liebig's Ann. Pharm., 13, 27. 
Wasser derart langsam, daß dieselbe im Vergleiche zu obiger Reaktion vollständig zu vernachlässigen ist. Man kann also den zeitlichen Fortschritt der Reaktion durch Bestimmung des Säuretiters der Reaktionsflüssigkeit zu verschiedenen Zeiten, der ja der gebildeten Menge Äthylschwefelsäure proportional ist, messend verfolgen.

Nur ist zu bemerken, daß infolge der Schwerlöslichkeit von Diäthylsulfat sowie von Dimethylsulfat in Wasser die Reaktion eine solche im heterogenen System ist und man, um gut vergleichbare Resultate $z u$ erhalten, für stets gleichmäßige Durchmischung der Reaktionsflüssigkeit zu sorgen hat;

Da einmal der eine reagierende Stoff im Überschusse vorhanden war, zum zweiten eine Reaktion im heterogenen System vorliegt, so müssen die Werte der Reaktionsgeschwindigkeit nach der Formel, wie sie für monomolekulare Reaktion gilt:

$$
k=\frac{1}{t} \ln \frac{a}{a-x}
$$

berechnet werden.

Hier zeigt sich nun ein eigentümliches Verhalten. Es war zu erwarten, daß die Werte von $k$ nicht, wie beobachtet wurde, bis gegen Ende der Reaktion konstant bleiben, sondern allmählich zunehmen würden. Denn es war anzunehmen, daß die während der Reaktion gebildeten $\mathrm{H}$-Ionen, die bei der relativen Stärke der Äthylschwefelsäure ${ }^{1}$ sich in reichem Maße bilden müssen, die Abspaltung der ersten Alkylgruppe durch Wasser katalytisch beschleunigen würden.

Die Tatsache, daß die Werte von $k$ bis gegen Ende der Reaktion konstant sind, ließ vermuten, daß bei del behandelten Reaktion unter den gegebenen Umständen Wasserstoff-Ionen eine katalytische Beschleunigung nicht verursachen. Versuche, bei denen stait Wasser 2·0-, 1·0-, $0 \cdot 4$ normale Schwefelsäure zur Abspaliung der ersten Alkylgruppe aus Diäthylsulfat und Dimethylsulfat verwendet wurden, ergaben die Richtigkeit dieser Vermutung. Der Säurezusatz wirkt hier nämlich nicht nur nicht reaktionsbeschleunigend, sondern bei den höheren Säurekonzentrationen sogar verzögernd.

1 Ostwald, Zeitschr. für physik. Chemie, 1, 76, 81 . 
Dieser Umstand, daß H-Ionen bei Esterverseifung nicht als positive Katalysatoren wirken, ist nicht ganz neu. Vor kurzer Zeit erst zeigte Praetorius im Wegscheider'schen Institut, daß die Verseifung des Benzolsulfosäuremethylesters durch H-Ionen nicht beschleunigt wird. ${ }^{1}$

$\mathrm{Ob}$ aber die von mir beobachtete katalytische Unwirksamkeit, beziehungsweise Verzögerung auf die spezifische Natur der untersuchten Ester zurückzuführen ist, was bei der analogen Konstitution des von Praetorius untersuchten Esters mit den von mir angewandten Estern nicht überraschen könnte oder aber hier eine Eigentümlichkeit des heterogenen Systems ist, hervorgerufen durch Änderung der Diffusionsgeschwindigkeit beim Übergange von Wasser zu Schwefelsäuren verschiedener Konzentrationen, kann im Hinblick auf die Schwerlöslichkeit der Ester schwer entschieden werden. Allerdings tritt bei der Verseifung durch Alkali eine bedeutende, der Alkalikonzentration proportionale Steigerung der Reaktionsgeschwindigkeit ein, obgleich auch hier heterogenes System vorliegt und man Änderung der Diffusionsgeschwindigkeit beim Übergange von Wasser zu Alkali im selben Sinn annehmen muß wie beim Übergange von Wasser zu Schwefelsäure.

Als statt Schwefelsäure Salzsäure verwendet wurde, beobachtete ich eine analoge Nebenreaktion, wie sie von Praetorius bei der Verseifung von Benzolsulfonsäureester durch Salzsäure beobachtet wurde. Es reagiert nämlich Diäthylsulfat mit Salzsäure unter Bildung von Chloräthyl nach der Gleichung

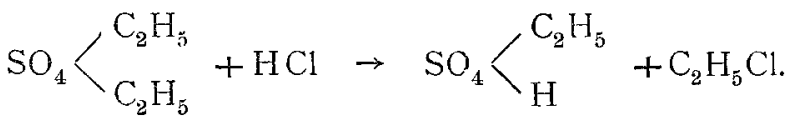

Wegen der kinetisch messenden Verfolgung dieser Reaktion sei auf den experimentellen Teil verwiesen. Hervorgehoben sei nur, daß auch hier eine Beschleunigung der oben erwähnten Reaktion der Verseifung von Diäthylsulfat durch Wasser nicht statthat und daß die Geschwindigkeit der Bildung von Chloräthyl merklich größer ist, schon in 2 norm. $\mathrm{HCl}$, als die Verseifung von Diäthylsulfat durch Wasser.

1 Monatshefte für Chemie, 26, 1 (1905). 
Einen ganz analogen "Esteraustausch" beobachte ich auch bei Verwendung von $\mathrm{HNO}_{3}$ als Katalysator. Wie zu erwarten, ist in beiden Fällen die Geschwindigkeit der Bildung der Ester der zugesetzten Säuren deren Konzentration proportional.

Bemerkenswert ist der abnorme Temperaturkoeffizient der Reaktion der Abspaltung der ersten Alkylgruppe aus Dialkylsulfaten. Es beträgt pro $10^{\circ}$ rund 4 , die Reaktionsgeschwindigkeit vervierfacht sich pro $10^{\circ}$, während in der Regel bei den meisten chemischen Reaktionen Verdoppelung der Reaktionsgeschwindigkeit pro $10^{\circ}$ eintritt.

Ich bin geneigt, diese Abnormität als Eigenheit des heterogenen Systems anzusehen. Denn viele Reaktionen, bei denen Gasabscheidung eintritt, die also gleichfalls heterogenes System repräsentieren, zeigen Temperaturkoeffizienten von über 4 pro $10^{\circ} .^{1}$

Es dürfte also die verbreitete Meinung, daß physikalische Vorgänge gegenüber chemischen abnorm kleine Temperaturkoeffizienten aufweisen, ${ }^{2}$ nicht immer zutreffend sein, denn in heterogenen Systemen sind ja für die Reaktionsgeschwindigkeit vor allem physikalische Vorgänge mâjgebend.

\section{Experimenteller Teil.}

\section{Darstellung der verwendeten Dialkylsulfate und Verseifung derselben durch Wasser bei verschiedenen Temperaturen.}

Das verwendete Diäthylsulfat wurde nach der Vorschrift von Wetherill ${ }^{3}$ dargestellt und durch fraktionierte Destillation im Vakuum gereinigt.

Das angewandte Dimethylsulfat wurde von Kahlbaum bezogen und gleichfalls der fraktionierten Destillation im Vakuum unterworfen.

1 Goldschmidt H. und Bräuer R., Chem. Ber. 39, p. 109 bis 112, Balcom, Inaug. Dis., Heidelberg 1905.

2 Siehe Stock und Guttmann, Chem. Ber., 37, 1959 (1906).

3 Lieb. Ann. der Chemie, 60, 117, 1848. 
Von den gereinigten Estern wurde mit einer in HundertstelKubikzentimeter geteilten Pipette, an der noch Tausendstel schätzbar waren, je $0.5 \mathrm{~cm}^{3}$ abgemessen und in ausgedämpften, $20 \mathrm{~cm}^{3}$ fassenden Fläschchen mit je $10 \mathrm{~cm}^{3}$ auf die Versuchstemperatur vorgewärmten Wassers gemischt und in einem Schüttelapparat, wie er in einer früheren Abhandlung eingehend beschrieben worden war, ${ }^{1}$ mit konstanter Schüttelgeschwindigkeit geschüttelt. In verschiedenen Zeitintervallen wurden nun die Fläschchen herausgenommen und der Säuretiter ermittelt, der in Kubikzentimeter $0 \cdot 1$ norm. Lösung in der zweiten Spalte der im folgenden mitgeteilten Tabellen der Versuchsergebnisse unter $x$ eingetragen ist und im Hinblick auf die geringe Verseifungsgeschwindigkeit von Äthylschwefelsäure unter den angewandten Bedingungen der gebildeten Menge dieser Säure proportional ist. In der dritten Spalte der Tabellen sind die zur Zeit $t$ (erste Spalte der Tabellen) nicht umgesetzten Mengen Diäthylsulfat gleichfalls in Kubikzentimeter 0.1 norm. Lösung eingetragen. Diese Werte $a-x$ sind aus der Differenz der umgesetzten Menge $x$ und dem Wert $a$, das ist die zur Zeit $t=\infty$ gebildete Menge Äthylschwefelsäure, gebildet. Die letzte Spalte der Tabellen gibt die Werte der Reaktionsgeschwindigkeit berechnet nach der Formel:

$$
k=\frac{1}{t} \ln \frac{a}{a-x} .
$$

Siehe die Tabellen 1, 2, 3, 4 .

Man sieht, die Werte von $k$ sind in Berücksichtigung der bei Untersuchungen im heterogenen System unvermeidlichen Fehler, die vielleicht durch Unregelmäßigkeit des Schüttelns verursacht werden, bis gegen Ende der Reaktion eine gute. Es ist also nicht anzunehmen, daß̧ die durch die Bildung von Äthylschwefelsäure vorhandenen H-Ionen die Reaktion merklich beeinflussen. Bemerkenswert ist auch der abnorm hohe Temperaturkoeffizient, der 3.5 bei Verseifung von Dimethylsulfat, der von Diäthylsulfat $4 \cdot 2$ pro $10^{\circ}$ beträgt.

1 Monatshefte für Chemie, 26, 315 (1905). 
R. Kremann,

Tabelle 1.

Verseifung von Dimethylsulfat mit Wasser bei $33.5^{\circ}$.

\begin{tabular}{|c|c|c|c|}
\hline $\begin{array}{c}t \\
\text { in Minuten }\end{array}$ & $x$ & $a-x$ & $k$ \\
\hline & 19.4 & 33.6 & 0.0079 \\
25 & $25 \cdot 3$ & 27.7 & 0.0083 \\
34 & 29.7 & 23.3 & 0.0084 \\
42 & 37.0 & 16.0 & 0.0080 \\
65 & 40.8 & 12.2 & 0.0080 \\
80 & 43.3 & 0.7 & 0.0082 \\
90 & 16.2 & 36.8 & 0.0077 \\
20.5 & 11.5 & 41.5 & 0.0076 \\
14.0 & 7.7 & 45.3 & 0.0080 \\
8.5 & 4.6 & $48 \cdot 4$ & 0.0079 \\
5.0 & 53.0 & 0.0 & \\
$\infty$ & & & \\
& & &
\end{tabular}

Tabelle 2 .

Verseifung von Diäthylsulfat mit Wasser bei $24 \cdot 0^{\circ}$.

\begin{tabular}{|c|c|c|c|}
\hline $\begin{array}{c}t \\
\text { in Minuten }\end{array}$ & $x$ & $a-x$ & $k$ \\
\hline 93 & $20 \cdot 4$ & $32 \cdot 6$ & 0.0023 \\
\hline 109 & $23 \cdot 8$ & $29 \cdot 2$ & 0.0024 \\
\hline 140 & $28 \cdot 6$ & $24 \cdot 7$ & 0.0024 \\
\hline 77 & $17 \cdot 2$ & $35 \cdot 8$ & $0 \cdot 0022$ \\
\hline 55 & $13 \cdot 0$ & $40 \cdot 0$ & 0.0022 \\
\hline 45 & $10 \cdot 6$ & $42 \cdot 4$ & 0.0022 \\
\hline 31 & $7 \cdot 6$ & $45 \cdot 4$ & 0.0022 \\
\hline 16 & $4 \cdot 6$ & $48 \cdot 4$ & 0.0025 \\
\hline 1505 & $31 \cdot 6$ & $21 \cdot 4$ & 0.0025 \\
\hline 190 & $35 \cdot 4$ & $17 \cdot 6$ & 0.0025 \\
\hline 200 & $36 \cdot 6$ & $16 \cdot 4$ & 0.0025 \\
\hline$\infty$ & $58 \cdot 0$ & 0.0 & \\
\hline
\end{tabular}


Tabelle 3.

Verseifung von Diäthylsulfat bei $34^{\circ}$.

\begin{tabular}{|c|c|c|c|}
\hline $\begin{array}{c}t \\
\text { in Minuten }\end{array}$ & $x$ & $a-x$ & $k$ \\
\hline 195 & $20 \cdot 6$ & $17 \cdot 4$ & $0 \cdot 0017$ \\
\hline 56 & $6 \cdot 8$ & $31 \cdot 2$ & $0 \cdot 0015$ \\
\hline 147 & $16 \cdot 55$ & $21 \cdot 35$ & 0.0017 \\
\hline 119 & $14 \cdot 1$ & $24 \cdot 7$ & 0.0016 \\
\hline 87 & $10 \cdot 3$ & $27 \cdot 7$ & 0.0016 \\
\hline 30 & $3 \cdot 8$ & $34 \cdot 2$ & 0.0015 \\
\hline 50 & $5 \cdot 9$ & $32 \cdot 1$ & 0.0015 \\
\hline 89 & $9 \cdot 4$ & $28 \cdot 6$ & 0.0014 \\
\hline 144 & $13 \cdot 7$ & $24 \cdot 3$ & 0.0014 \\
\hline$\infty$ & $38 \cdot 0$ & $0 \cdot 0$ & Mittel 0.00155 \\
\hline
\end{tabular}

Tabelle 4.

Verseifung von Diäthylsulfat mit Wasser bei $44^{\circ}$.

\begin{tabular}{|c|c|c|c|}
\hline $\begin{array}{c}t \\
\text { in Minuten }\end{array}$ & $x$ & $a-x$ & $k$ \\
\hline 40 & 16.6 & 21.4 & 0.0062 \\
95 & 29.6 & 8.4 & 0.0069 \\
69 & 24.5 & 13.5 & 0.0065 \\
20 & 10.2 & 27.8 & 0.0068 \\
50 & 20.2 & 17.8 & 0.0066 \\
$\infty$ & 38.0 & 0.0 & \\
& & &
\end{tabular}




\section{Verseifung von Dialkylsulfaten durch Schwefelsäure ver- schiedener Konzentrationen.}

Um die vermutete Unwirksamkeit von H-Ionen bei den behandelten Reaktionen festzustellen, unternahm ich die Verseifung statt mit Wasser mit Schwefelsäure von verschiedenen Konzentrationen $(2 \cdot 0-, 1 \cdot 0$ - und $0 \cdot 4$ normal).

Die Versuchsbedingungen waren die ganz gleichen wie bei der früher beschriebenen Verseifung durch Wasser, nur wurden statt $10 \mathrm{~cm}^{3}$ Wasser $10 \mathrm{~cm}^{3}$ auf die Versuchstemperatur vorgewärmter Schwefelsäure verwendet. In einzelnen Zeitintervallen wurden die einzelnen Fläschchen aus dem Schüttelapparat herausgenommen und die Reaktionsflüssigkeit in eine Lauge einfließen gelassen, die genau den $10 \mathrm{~cm}^{3}$ der zugegebenen Säure äquivalent war. Dann wurde der restliche Säuretiter mit $0 \cdot 1$ norm. Alkali bestimmt. Dieser entspricht dann wieder direkt der gebildeten Menge Äthylschwefelsäure in Kubikzentimeter $0 \cdot 1$ norm. Lösung.

Die so in einzelnen Zeitintervallen erhaltenen Werte sind dann in den folgenden Tabellen 5, 6, 7, 8, 9 in gleicher Weise eingetragen, wie bei der Wasserverseifung dargelegt wurde.

Tabelle 5 .

Verseifung von Diäthylsulfat mit 2norm. $\mathrm{H}_{2} \mathrm{SO}_{4}$ bei $44^{\circ}$.

\begin{tabular}{|c|c|c|c|}
\hline $\begin{array}{c}t \\
\text { in Minuten }\end{array}$ & $x$ & $a-x$ & $k$ \\
\hline 34 & 11.0 & 27.0 & 0.0044 \\
78.5 & 20.5 & 17.5 & 0.0043 \\
96.5 & 24.5 & 13.5 & 0.0047 \\
22.0 & 7.2 & 30.8 & 0.0042 \\
63.0 & 18.3 & 19.7 & 0.0045 \\
15.5 & 5.8 & 32.2 & 0.0046 \\
& & &
\end{tabular}


Tabelle 6.

Verseifung von Diäthylsulfat mit 1 norm. $\mathrm{H}_{2} \mathrm{SO}_{4}$ bei $44^{\circ}$.

\begin{tabular}{|c|c|c|c|}
\hline $\begin{array}{c}t \\
\text { in Minuten }\end{array}$ & $x$ & $a-x$ & $k$ \\
\hline \hline 90 & $23 \cdot 4$ & $14 \cdot 6$ & 0.0046 \\
60 & $18 \cdot 0$ & $20 \cdot 0$ & 0.0047 \\
47 & $15 \cdot 4$ & $22 \cdot 6$ & 0.0048 \\
19 & 6.8 & $31 \cdot 2$ & 0.0045 \\
15 & 5.6 & $32 \cdot 4$ & 0.0046 \\
\hline
\end{tabular}

Tabelle 7.

Verseifung von Diäthylsulfat mit 0.4 norm. $\mathrm{H}_{2} \mathrm{SO}_{4}$ bei $44^{\circ}$.

\begin{tabular}{|c|r|r|r|}
\hline $\begin{array}{c}t \\
\text { in Minuten }\end{array}$ & $x$ & $a-x$ & $k$ \\
\hline \hline 44 & 18.5 & 19.5 & 0.0065 \\
45 & 29.8 & 8.2 & 0.0070 \\
25 & 11.7 & 26.3 & 0.0065 \\
60 & 23.4 & 14.6 & 0.0070 \\
12 & 6.2 & 31.8 & 0.0065 \\
$\infty$ & 38.0 & 0.0 & \\
\hline
\end{tabular}

Tabelle 8.

Verseifung von Diäthylsulfat mit 1.0 norm. $\mathrm{H}_{2} \mathrm{SO}_{4}$ bei $36^{\circ}$.

\begin{tabular}{|c|c|c|c|c|}
\hline $\begin{array}{c}t \\
\text { in Minuten }\end{array}$ & $x$ & $a-x$ & $\begin{array}{c}k_{36} \\
\text { durch Säure }\end{array}$ & $\begin{array}{c}k_{36}^{\prime} \\
\text { durch Wasser }\end{array}$ \\
\hline \hline 72 & $9 \cdot 1$ & 28.4 & 0.0016 & \\
113 & 12.4 & 25.6 & 0.0015 & 0.0021 \\
141 & 14.6 & 23.4 & 0.0015 & \\
197 & 20.2 & 17.8 & 0.0016 & \\
$\infty$ & 38.0 & 0.0 & &
\end{tabular}


Tabelle 9.

Verseifung von Dimethylsulfat mit 1 norm. $\mathrm{H}_{2} \mathrm{SO}_{4}$ bei $24 \cdot 0^{\circ}$.

\begin{tabular}{|c|c|c|c|}
\hline $\begin{array}{c}t \\
\text { in Minuten }\end{array}$ & $x$ & $a-x$ & $k$ \\
\hline 126 & $24 \cdot 5$ & $28 \cdot 5$ & $0 \cdot 0021$ \\
\hline 101 & $20 \cdot 0$ & $33 \cdot 0$ & 0.0020 \\
\hline 80 & $15 \cdot 5$ & $37 \cdot 5$ & $0 \cdot 0019$ \\
\hline 60 & $12 \cdot 5$ & $40 \cdot 5$ & 0.0020 \\
\hline 44 & $9 \cdot 8$ & $43 \cdot 2$ & 0.0020 \\
\hline 29 & $7 \cdot 1$ & $45 \cdot 9$ & 0.0021 \\
\hline 18 & $4 \cdot 4$ & $48 \cdot 6$ & 0.0021 \\
\hline 131 & $25 \cdot 1$ & $27 \cdot 9$ & 0.0021 \\
\hline 177 & $30 \cdot 6$ & $22 \cdot t$ & 0.0021 \\
\hline 197 & $32 \cdot 7$ & $20 \cdot 3$ & 0.0021 \\
\hline$\infty$ & $53 \cdot 0$ & 0.0 & \\
\hline
\end{tabular}

Man sieht aus den Tabellen, daß Schwefelsäure in Konzentration von 0.4 normal die Reaktionsgeschwindigkeit nicht beeinflußt, in höheren Konzentrationen, 1.0- und $2 \cdot 0$ normal, eine merkliche Verzögerung der Verseifungsgeschwindigkeit bewirkt.

\section{Verseifung von Diäthylsulfat durch Alkali.}

In ganz analoger Weise wie die Verseifung durch Wasser und Schwefelsäure wurde auch die mit Alkali vorgenommen. Je $0.5 \mathrm{~cm}^{3}$ des Esters wurden mit $10 \mathrm{~cm}^{3}$ des Alkalis von bestimmter Konzentration, das auf die Versuchstemperatur vorgewärmt war, vermischt, eine Zeit $t$ lang geschüttelt und dann die Reaktionsflüssigkeit in Säure einfließen gelassen, die den $10 \mathrm{~cm}^{3}$ angewandten Alkalis genau äquivalent war, und dann der restliche Säuretiter, der der gebildeten Menge Äthylschwefelsäure entsprach, mit $0 \cdot 1$ norm. Alkali bestimmt. So haben die Werte in den einzelnen Tabellen 10 und 11 die gleiche Bedeutung wie in den früher mitgeteilten Tabellen. 
Tabelle 10.

Verseifung von Diäthylsulfat mit 0.4 norm. $\mathrm{NaOH}$ bei $34^{\circ}$.

\begin{tabular}{|c|c|c|c|}
\hline $\begin{array}{c}t \\
\text { in Minuten }\end{array}$ & $x$ & $a-x$ & $k$ \\
\hline 123 & 28.8 & 10.8 & 0.0044 \\
40 & $12 \cdot 7$ & $25 \cdot 3$ & 0.0044 \\
59 & $17 \cdot 5$ & $20 \cdot 5$ & 0.0045 \\
97 & 24.6 & 13.4 & 0.0047 \\
80 & 21.2 & 16.3 & 0.0044 \\
30 & 10.4 & 27.6 & 0.0046 \\
47 & 14.5 & 23.5 & 0.0044 \\
$\infty$ & 38.0 & 0.0 & \\
& & &
\end{tabular}

Tabelle 11.

Verseifung von Diäthylsulfat mit 1.0 norm. $\mathrm{NaOH}$ bei $34^{\circ}$.

\begin{tabular}{|c|c|c|c|}
\hline $\begin{array}{c}t \\
\text { in Minuten }\end{array}$ & $x$ & $a-x$ & $k$ \\
\hline \hline 12 & 9.1 & 28.9 & 0.0099 \\
27 & $16 \cdot 8$ & $21 \cdot 2$ & 0.0094 \\
45 & 24.6 & $13 \cdot 4$ & 0.0098 \\
20 & 12.8 & $25 \cdot 2$ & 0.0090 \\
$\infty$ & 38.0 & 0.0 &
\end{tabular}

Wie man sieht, ist die Geschwindigkeit der alkalischen Verseifung bedeutend größer als die der wässerigen Verseifung durch Wasser allein, und zwar ziemlich gut proportional der Alkalikonzentration. 


\section{Verseifung von Diäthylsulfat durch Salzsäure von ver- schiedener Konzentration.}

Die Versuchsanordnung war die gleiche wie bei der Verseifung durch Schwefelsäure. Es wurden wieder je $0.5 \mathrm{~cm}^{3}$ Diäthylsulfat mit je $10 \mathrm{~cm}^{3}$ auf die Versuchstemperatur vorgewärmte Salzsäure in Konzentrationen von $2 \cdot 0-, 1 \cdot 0$ - und 0.4 normal in einzelnen Fläschchen zusammengebracht und im Schüttelapparat geschüttelt. Dann wurde die Reaktionsflüssigkeit wieder in Lauge einfließen gelassen, die gerade den $10 \mathrm{~cm}^{3}$ zugesetzter Salzsäure äquivalent war und der Säureüberschuß mit 0.1 norm. Alkali titriert, der nun wieder der gebildeten Äthylschwefelsäure proportional sein sollte und in Kubikzentimeter 0.1 norm. Lösung in der zweiten Spalte der folgenden Tabelle 12 eingetragen ist.

Es stellte sich nun heraus, daß hier als Endtiter der Reaktion nicht der bei Verseifung durch Schwefelsäure, Wasser und Alkali $0.5 \mathrm{~cm}^{3}$ Diäthylsulfat entsprechende Wert von $38 \mathrm{~cm}^{3} \mathrm{0} \cdot 1$ norm. Lösung ermittelt wurde, sondern mit zunehmender Konzentration der Salzsäure immer kleiner werdende Werte als Endtiter der Reaktion gefunden wurden.

In der folgenden Tabelle 12 sind die Endtiter in ihrer Abhängigkeit von der Salzsäurekonzentration ersichtlich. In der ersten Spalte sind die Salzsäurekonzentrationen in Normalitäten, in der zweiten Spalte die Endtiter verzeichnet.

Dieser Umstand läßt sich auf die Tatsache zurückführen, daß neben der Verseifung durch Wasser nach

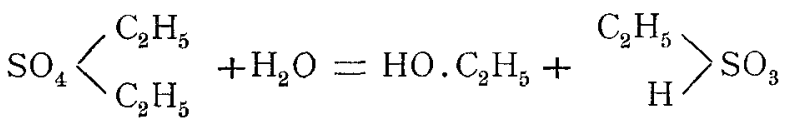

Salzsäure gleichfalls mit Diäthylsulfat, und zwar unter Bildung von Chloräthyl nach

$$
\mathrm{SO}_{4}\left\langle\begin{array}{l}
\mathrm{C}_{2} \mathrm{H}_{5} \\
\mathrm{C}_{2} \mathrm{H}_{5}
\end{array}+\mathrm{HCl}=\mathrm{ClC}_{2} \mathrm{H}_{5}+\stackrel{\mathrm{C}_{2} \mathrm{H}_{5}}{\mathrm{H}^{\prime}}\right\rangle \mathrm{SO}_{4}
$$

reagiert. Das entstandene Chloräthyl machte sich durch Geruch und hohen Druck in den Fläschchen bemerkbar und konnte als farblose Flüssigkeit vom Siedepunkt $12 \cdot 5^{\circ}$ isoliert werden. 
Wirkung von H-Ionen.

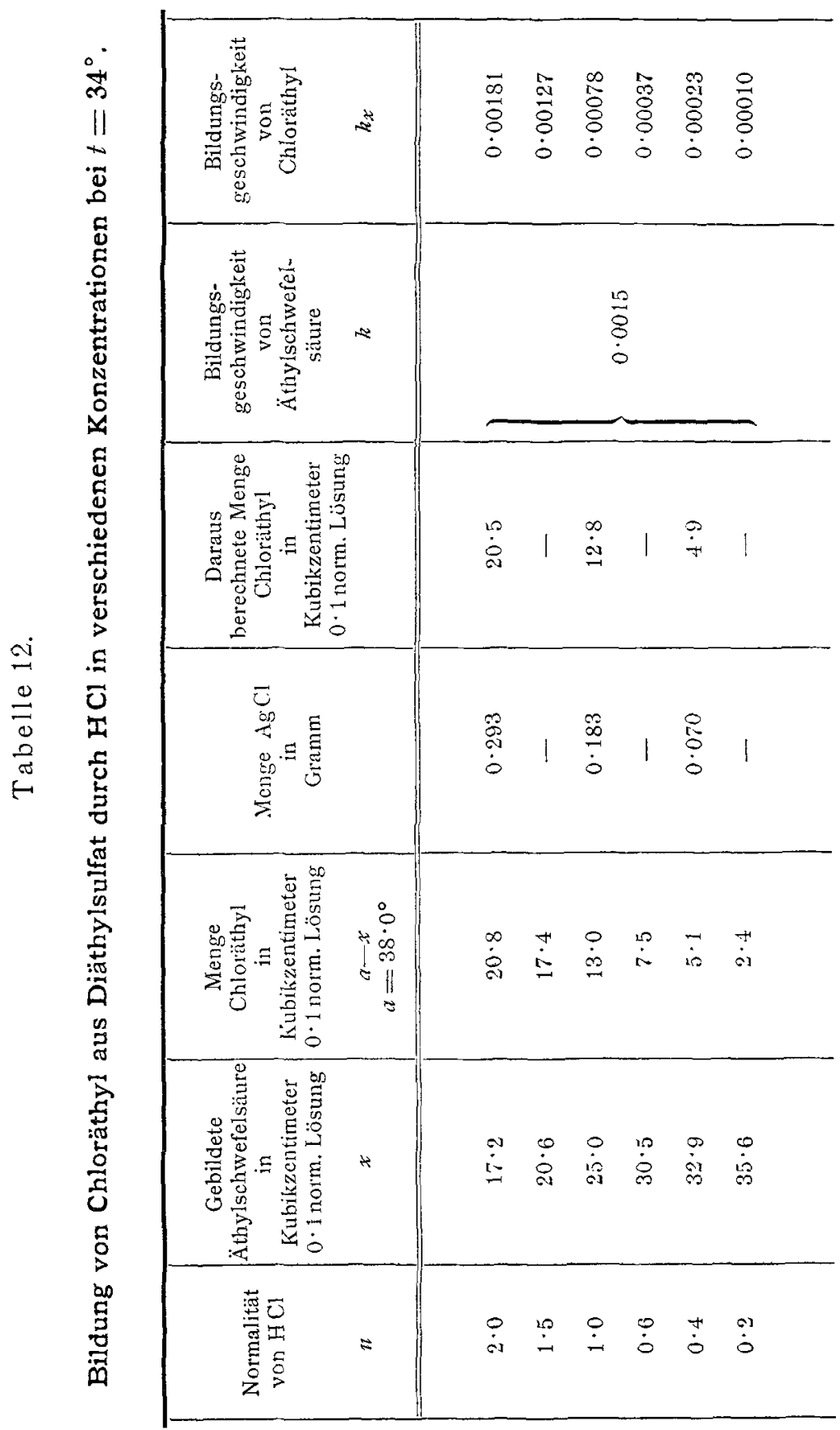


$0.573 \mathrm{~g}$ Substanz gaben $1.265 \mathrm{~g} \mathrm{AgCl}=0.314 \mathrm{~g} \mathrm{Cl}$.

In 100 Teilen:

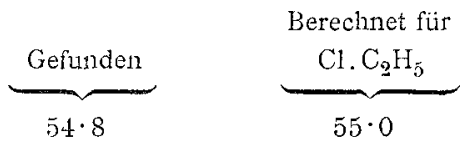

Äthylschwefelsäure wird bei der Verseifung von Diäthylsulfat durch $\mathrm{HCl}$ durch zwei verschiedene Reaktionen gebildet, nach Gleichung (1) und nach Gleichung (2). Eine einfache Überlegung lehrt jedoch, daß die durch Titration mit 0.1 norm. Alkali nach Abstumpfung der ursprünglich zugegebenen Salzsäure durch die genau äquivalente Menge Alkali, wie es der Versuchsanordnung entsprach, lediglich der nach Gleichung (1), also der Verseifung durch Wasser gebildeten Menge Äthylschwefelsäure entsprechen. Denn für jedes Mol Äthylschwefelsäure, die durch die Reaktion mit $\mathrm{HCl}$, also nach Gleichung (2), gebildet wird, wird die äquivalente Menge $\mathrm{HCl}$ infolge der Bildung von Chloräthyl entzogen, so daß, falls nur die Reaktion mit Salzsäure, also lediglich Gleichung (2), von statten ginge, überhaupt keine Änderung des Säuretiters wahrnehmbar wäre. Es ist also die Zunahme des Säuretiters einzig und allein durch die Verseifung mit Wasser, also die Reaktion nach Gleichung (2), bedingt.

Die in den folgenden Tabellen $13,14,15,16,17$ in der zweiten Spalte mitgeteilten Werte $x$ sind die in Kubikzentimeter 0.1 norm. Lösung ausgedrückten Mengen Äthylschwefelsäure, wie sie durch Verseifung von Diäthylsulfat durch Wasser entstehen. Wan sieht, daß auch hier die H-Ionen nicht katalytisch beschleunigend wirken, wenn auch eine katalytische Verzögerung nicht zu beobachten ist. Will man einen Einblick in die Geschwindigkeit der Chloräthylbildung, der Gleichung (2), gewinnen, so kann man sich folgender Überlegung bediencn.

$38 \mathrm{~cm}^{3} 0.1$ norm. Äthylschwefelsäure können aus $0.5 \mathrm{~cm}^{3}$ Diäthylsulfat gewonnen werden. Die durch Verseifung mit Wasser gebildete Menge Äthylschwefelsäure kann nach früher Dargelegtem durch Zunahme des Säuretiters der Reaktionsflüssigkeit bestimmt werden. 
Tabelle 13.

Verseifung von Diäthylsulfat mit $2 \cdot 0$ norm. $\mathrm{HCl}$ bei $44^{\circ}$.

\begin{tabular}{|c|c|c|c|}
\hline $\begin{array}{c}t \\
\text { in Minuten }\end{array}$ & $x$ & $a-x$ & $k$ \\
\hline 31 & 6.5 & 11.6 & 0.0062 \\
12 & 3.0 & $15 \cdot 1$ & 0.0066 \\
46 & 9.4 & $8 \cdot 7$ & 0.0069 \\
79 & 12.7 & $5 \cdot 4$ & 0.0067 \\
110 & 15.0 & 3.1 & 0.0070 \\
$\infty$ & 18.1 & 0.0 &
\end{tabular}

Tabelle 14.

Verseifung von Diäthylsulfat mit 1.0 norm. $\mathrm{HCl}$ bei $44^{\circ}$.

\begin{tabular}{|c|c|c|c|}
\hline $\begin{array}{c}t \\
\text { in Minuten }\end{array}$ & $x$ & $a-x$ & $k$ \\
\hline \hline 15 & 5.2 & 18.8 & 0.0071 \\
30 & 8.8 & 15.2 & 0.0067 \\
48 & 12.6 & 11.4 & 0.0067 \\
75 & 16.6 & 7.4 & 0.0068 \\
$\infty$ & 24.0 & 0.0 & \\
\hline
\end{tabular}

Tabelle 15.

Verseifung von Diäthylsulfat mit 1.0 norm. $\mathrm{HCl}$ bei $36^{\circ}$.

\begin{tabular}{|c|c|c|c|}
\hline $\begin{array}{c}t \\
\text { in Minuten }\end{array}$ & $x$ & $a-x$ & $k$ \\
\hline 137 & $12 \cdot 1$ & $12 \cdot 9$ & 0.0021 \\
\hline 195 & $15 \cdot 9$ & $9 \cdot 1$ & 0.0023 \\
\hline 400 & $22 \cdot 4$ & $2 \cdot 6$ & 0.0025 \\
\hline 320 & $20 \cdot 9$ & $4 \cdot 1$ & 0.0025 \\
\hline 263 & $19 \cdot 7$ & $5 \cdot 3$ & 0.0026 \\
\hline 153 & $13 \cdot 8$ & $11 \cdot 2$ & 0.0023 \\
\hline 88 & $8 \cdot 8$ & $16 \cdot 2$ & 0.0021 \\
\hline 57 & $6 \cdot 8$ & $18 \cdot 2$ & 0.0024 \\
\hline 27 & $3 \cdot 4$ & $21 \cdot 6$ & 0.0024 \\
\hline$\infty$ & $25 \cdot 0$ & 0.0 & \\
\hline
\end{tabular}


R. Kremann,

Tabelle 16.

Verseifung von Diäthylsulfat mit 1.0 norm. $\mathrm{HCl}$ bei $34^{\circ}$.

\begin{tabular}{|c|c|c|c|}
\hline $\begin{array}{c}t \\
\text { in Minuten }\end{array}$ & $x$ & $a-x$ & $k$ \\
\hline 56 & 4.1 & $20 \cdot 9$ & $0 \cdot 0014$ \\
87 & 6.3 & $18 \cdot 7$ & 0.0014 \\
149 & $9 \cdot 7$ & $15 \cdot 3$ & 0.0014 \\
$\infty$ & $25 \cdot 0$ & $0 \cdot 0$ & \\
\hline
\end{tabular}

Tabelle 17 .

Verseifung von Diäthylsulfat mit 0.4 norm. $\mathrm{HCl}$ bei $35^{\circ}$.

\begin{tabular}{|c|c|c|c|}
\hline $\begin{array}{c}t \\
\text { in Minuten }\end{array}$ & $x$ & $a-x$ & $k$ \\
\hline \hline 60 & $7 \cdot 1$ & $25 \cdot 8$ & 0.0018 \\
92 & $10 \cdot 4$ & 22.5 & 0.0018 \\
118 & 12.8 & $20 \cdot 1$ & 0.0018 \\
150 & 15.6 & $17 \cdot 3$ & 0.0019 \\
184 & 18.1 & 14.8 & 0.0019 \\
28 & 3.8 & 29.1 & 0.0019 \\
$\infty$ & 32.9 & 0.0 &
\end{tabular}

Wäre z. B. bei irgend einer Salzsäurekonzentration bei der Konkurrenz von $\mathrm{HCl}$ und $\mathrm{H}_{2} \mathrm{O}$ um das vorhandene Diäthylsulfat die Geschwindigkeit der beiden Vorgänge gleich groß, so würden $38 / 2=19 \mathrm{~cm}^{3}$ Äthylschwefelsäure durch die Reaktion mit Wasser gebildet werden und $38-19=19 \mathrm{~cm}^{3}$ durch die Reaktion mit Salzsäure. Diese $19 \mathrm{~cm}^{3}$ würden natürlich auch 
der gebildeten Menge Chloräthyl entsprechen. Ganz allgemein: Ist $x$ die Anzahl Kubikzentimeter durch Wasser gebildeter Äthylschwefelsäure, so sind $(38-x) \mathrm{cm}^{3}$ die Anzahl Kubikzentimeter $0 \cdot 1$ norm. Äthylschwefelsäure, die durch die Reaktion mit Salzsäure entstanden sind und zugleich die Menge gebildeten Chloräthyls in Kubikzentimeter 0.1 norm. Lösung. Ist nun $k$ die Verseifungsgeschwindigkeit von Diäthylsulfat bei der bestimmten Versuchstemperatur, wie sie experimentell ermittelt werden kann, $k_{x}$ die $\mathrm{zu}$ bestimmende Bildungsgeschwindigkeit von Chloräthyl, so sind dieselben proportional der Anzahl Kubikzentimeter 0.1 norm. Lösung Äthylschwefelsäure, wie sie durch Wasserverseifung gebildet wird und durch Zunahme des Säuretiters der Reaktionsflüssigkeit ermittelt werden kann und dem Wert $(38-x) \mathrm{cm}^{3}$, der die Menge des gebildeten Chloräthyls darstellt. Es ist also

$$
\begin{aligned}
k: k_{x} & =x: 38-x, \\
k_{x} & =\frac{k(38-x)}{x} .
\end{aligned}
$$

Um darzutun, daß in der Tat $(38-x) \mathrm{cm}^{3}$ der Menge gebildeten Chloräthyls entsprach, wurde bei einzelnen Versuchen das gebildete Chloräthyl besonders bestimmt.

Es wurde durch die in den Fläschchen befindliche Reaktionsflüssigkeit, nachdem alles Diäthylsulfat reagiert hatte, Luft durchgesaugt. Diese Luft strich durch ein kleines, mit Kalkstücken gefülltes U-Röhrchen, um eventuell mitgerissene Salzsäure zurückzuhalten und wurde dann über rotglühenden, chlorfreien Kalk geleitet, wobei das mitgeführte Chloräthyl zerlegt wurde. Aus der gefundenen Chlormenge läßt sich dann die Menge Chloräthyl ermitteln. Die so gefundenen Werte, die in Tabelle 12, in der vierten Spalte eingetragen sind, stimmen mit den Differenzwerten $a-x$ gut überein. In der fünften Spalte der Tabelle 12 ist der Wert der Bildungsgeschwindigkeit von Äthylschwefelsäure durch Wasser als $k$, als Mittelwert der verschiedenen Bestimmungen mit 0.0015 eingetragen. In der letzten Spalte dieser Tabelle sind die Werte der Bildungsgeschwindigkeit von Chloräthyl, wie sie sich nach Gleichung (3) berechnen, als $k_{x}$ eingetragen. 
Wie die Theorie es verlangt, ist die Bildungsgeschwindigkeit nahezu gerade proportional der Konzentration der angewandten Salzsäure, wie beistehende graphische Darstellung es deutlich zeigt.

Bemerkenswert ist, daß, wie sich extrapolatorisch schätzen läßt, schon in einer 1.75 norm. $\mathrm{HCl}$ die Bildung von Chlor-

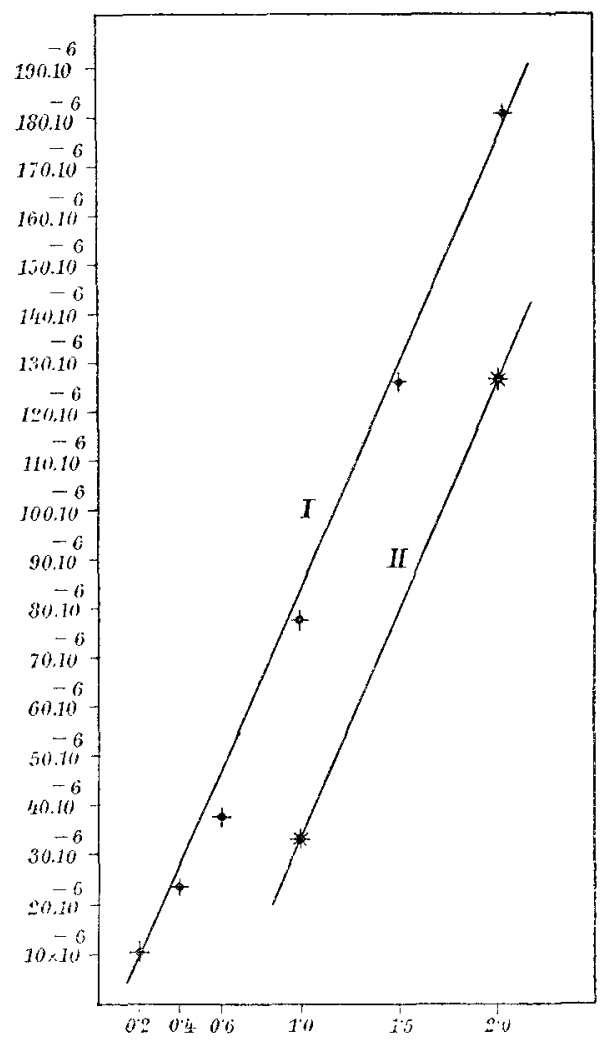

äthyl und die Zersetzung von Diäthylsulfat mit Wasser von gleicher Geschwindigkeit sind.

Ganz analog sind die Verhältnisse bei Salpetersäure. Die Versuchsanordnung war die gleiche. Auch in diesem Fall ist der Endtiter kleiner als $38 \mathrm{~cm}^{3} 0 \cdot 1$ norm. Lösung. Er nimmt mit steigender Konzentration der angewandten Säure ab, was sich in analoger Weise wie diese Abweichungen bei Salzsäure 
durch Chloräthylbildung aus Diäthylsulfat und Salzsäure, durch Bildung von Äthylnitrat aus Diäthylsulfat und Salpetersäure erklären lassen dürfte.

Folgende Tabelle 18 gibt die Versuchsergebnisse wieder.

Tabelle 18.

Bildung von Äthylnitrat aus Diäthylsulfat durch $\mathrm{HNO}_{3}$ in verschiedenen Konzentrationen bei $t=35 \cdot 0^{\circ}$.

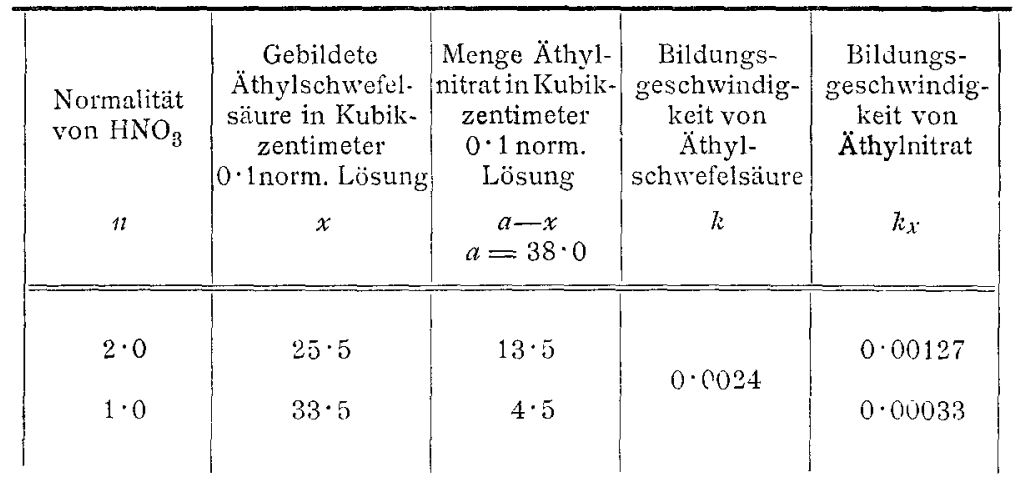

Die erste Spalte bedeutet die Normalität der Salpetersäure. In der zweiten ist die Menge der durch Verseifung durch Wasser gebildeten Äthylschwefelsäure in Kubikzentimeter 0.1 norm. Lösung, wie sie durch Zunahme des Säuretiters der Reaktionsflüssigkeit ermittelt wurde, verzeichnet. Die dritte Spalte gibt die Menge von gebildetem Äthylnitrat in Kubikzentimeter 0.1 norm. Lösung wieder, erhalten aus der Differenz von $a=38 \mathrm{~cm}^{3}$, der maximal möglich bildbaren Menge Äthylschwefelsäure und den Werten $x$ der zweiten Spalte. In der vierten Spalte ist der Wert der Verseifungsgeschwindigkeit von Diäthylsulfat durch $\mathrm{HNO}_{3}$ als Mittelwert aus den im folgenden mitgeteilten Versuchsergebnissen (Tabelle 19 und 20) verzeichnet. Die Werte der letzten Spalte $k$ sind die nach Irüher erwähnter Gleichung (3) berechneten Werte der Bildungsgeschwindigkeit von Äthylnitrat.

Man sieht aus der Figur (Linie II), daß auch hier die analoge Proportionalität zwischen Säurekonzentration und Bildungsgeschwindigkeit von Äthylnitrat statthat wie bei Chloräthyl- 
bildung. Nur ist in der Konkurrenz um Diäthylsulfat die Salpetersäure schwächer als die Salzsäure, da selbst in 2 norm. $\mathrm{HNO}_{3}$ noch immer die Wasserzersetzung des Diäthylsulfats mit größerer Geschwindigkeit von statten geht.

Wie schon früher erwähnt, kann die Verseifung von Diäthylsulfat durch Salpetersäure durch Bestimmung der Zunahme des Säuretiters messend verfolgt werden.

Folgende Tabellen geben die Versuchsergebnisse wieder. Tabelle 19.

Verseifung von Diäthylsulfat durch 1.0 norm. $\mathrm{HNO}_{3}$ bei $35^{\circ}$.

\begin{tabular}{|c|c|c|c|}
\hline $\begin{array}{c}t \\
\text { in Minuten }\end{array}$ & $x$ & $a-x$ & $k$ \\
\hline \hline 61 & $8 \cdot 5$ & $25 \cdot 0$ & 0.0021 \\
109 & 13.1 & 20.4 & 0.0019 \\
157 & 17.6 & 15.9 & 0.0021 \\
250 & 23.4 & 10.1 & 0.0021 \\
370 & 27.9 & 5.6 & 0.0021 \\
$\infty$ & 33.5 & 0.0 & \\
& & &
\end{tabular}

Tabelle 20 .

Verseifung von Diäthylsulfat durch $2 \cdot 0$ norm. $\mathrm{HNO}_{3}$ bei $35^{\circ}$.

\begin{tabular}{|c|c|c|c|}
\hline $\begin{array}{c}t \\
\text { in Minuten }\end{array}$ & $x$ & $a-x$ & $k$ \\
\hline \hline & $7 \cdot 7$ & $17 \cdot 3$ & 0.0026 \\
64 & $11 \cdot 8$ & $13 \cdot 2$ & 0.0024 \\
$117 \cdot 5$ & $16 \cdot 3$ & $8 \cdot 7$ & 0.0028 \\
164 & $18 \cdot 7$ & $6 \cdot 3$ & 0.0027 \\
233 & $25 \cdot 0$ & & \\
\hline
\end{tabular}

Wie man sieht, ist hier eine geringe Beschleunigung der Reaktion wahrzunehmen, gegenüber der Verseifung durch reines Wasser, proportional der Säurekonzentration. Doch spielt möglicherweise die sekundäre Natur des Anions hier mit, da bei beiden anderen untersuchten Säuren, der Salzsäure und der Schwefelsäure, eine solche Beschleunigung nicht zu konstatieren war. 